\title{
Ocular and Systemic Results of Intravitreal Bevacizumab Injection in Retinopathy of Prematurity Treatment
}

\author{
(D) Sadik Gorkem Cevik, ${ }^{1}$ (1) Mediha Tok Cevik, ${ }^{2}$ (1) Irfan Perente ${ }^{1}$ \\ ${ }^{1}$ Department of Ophthalmology, Yuksek Ihtisas Training and Research Hospital, Bursa,Turkey \\ ${ }^{2}$ Department of Opthalmology, Sisli Etfal Training and Research Hospital, Istanbul,Turkey
}

\begin{abstract}
Objectives: The aim of this study was to evaluate the effectiveness and safety of an intravitreal bevacizumab injection (IVB) in the treatment of retinopathy of prematurity (ROP).

Methods: The medical records of patients who had received IVB treatment for ROP between January 2014 and October 2018. Anatomical and functional outcomes were evaluated. The Denver II Developmental Screening Test was administered and fluorescein angiography (FA) was performed in some cases.

Results: Thirty-eight eyes of 19 infants were included in the study. An IVB injection was administered to 9 infants with aggressive posterior ROP (APROP) disease (Group I), 6 infants with any stage ROP with plus disease in zone I (Group 2), and 4 infants with stage 2-3 ROP with plus disease in zone II (Group 3). Complete retinal vascularization was observed in 24 eyes of 12 infants who received a single dose of bevacizumab without any additional treatment. Recurrence of the disease was observed in 12 eyes of 6 infants diagnosed with APROP and laser photocoagulation was performed. FA was performed to 5 IVB patients whose parents approved the procedure. The Denver II Developmental Screening Test was administered to all of the participants, and the test outcomes were consistent with the corrected age of the children, though 2 infants demonstrated a developmental delay in gross motor development tasks. Overall, good anatomical and functional results were obtained.

Conclusion: IVB is an effective and relatively safe treatment modality for infants with ROP; however, prospective studies are required to provide more detailed information about systemic side effects.

Keywords: Bevacizumab, Denver II, neurodevelopment, retinopathy of prematurity, safety.
\end{abstract}

\section{Introduction}

Retinopathy of prematurity (ROP) is an important cause of childhood blindness worldwide. The incidence of ROP has increased as the survival rate of premature infants with a lower gestational age increased (I). When an infant is born with incomplete vascularization, the retina tries to complete vascularization in an external environment. This can lead to the development of retinopathy. As the incidence of ROP has increased, studies related to treatment options and re- search demonstrating the effectiveness of peripheral avascular retina ablation in the treatment of ROP have multiplied $(2,3)$. The primary purpose of this treatment is to destroy and remove the areas of avascular retina to achieve regression of disease. However, since peripheral ablation causes destruction of a wide area of the retina, especially in zone I disease, new treatment modalities have been investigated (3, 4-9).

Once the role of vascular endothelial growth factor (VEGF) in the ROP etiopathogenesis was defined, treatment

Address for correspondence: Sadik Gorkem Cevik, MD. Yuksek Ihtisas Egitim ve Arastirma Hastanesi, Oftalmoloji Klinigi, Bursa, Turkey

Phone: +90 5077467970 E-mail: gorkemcevik@hotmail.com

Submitted Date: May 01, 2019 Accepted Date: March 07, 2020 Available Online Date: July 29, 2020

${ }^{\circ}$ Copyright 2020 by Beyoglu Eye Training and Research Hospital - Available online at www.beyoglueye.com OPEN ACCESS This work is licensed under a Creative Commons Attribution-NonCommercial 4.0 International License. 
with intravitreal injection of anti-VEGF drugs emerged. Bevacizumab is a humanized anti-VEGF monoclonal antibody commonly used in the treatment of $\operatorname{ROP}(4,5)$. An intact blood-retinal barrier is of utmost importance; hence, ROP infants often carry the risk of systemic absorption as a result of an impaired or underdeveloped barrier. The systemic and local side effects of anti-VEGF drugs and a precise dosage for ROP treatment in premature infants are still unknown $(6,7)$.

The primary purpose of this study was to evaluate the local and systemic effects of IVB in ROP treatment.

\section{Methods}

The medical records of patients who had undergone IVB treatment for ROP between January 2014 and October 2018 were reviewed. The Denver II Developmental Test was administered during control examinations after 2016 in order to evaluate and understand the systemic effects of IVB. The ROP classification and treatment and retreatment decisions were made according to the results of the international classification of ROP and Early Treatment for Retinopathy of Prematurity (ETROP) study (8-9).

This study was conducted in accordance with the principles of the Declaration of Helsinki and the study was approved by the hospital ethics committee.

Before the examination, the pupil was dilated with I.25\% phenylephrine (Mydfrin; Alcon, Geneva, Switzerland) and I\% tropicamide (Tropamid; Bilim Pharmaceuticals, Istanbul, Turkey). All of the examinations were performed using indirect ophthalmoscopy (Omega 500; Heine, Gilching, Germany) and the scleral indentation was performed by a single ophthalmologist. The exclusion criteria included a follow-up time of fewer than 18 months and the presence of any congenital ocular anomaly.

The IVB injection was performed in an operating room. Vital signs were monitored by an anesthesiology specialist during the entire procedure. Intravitreal injections were administered under topical anesthesia with $0.5 \%$ proparacaine hydrochloride ophthalmic solution (Alcain; Alcon, Geneva, Switzerland). After disinfection of the periocular skin and the conjunctiva with $2.5 \%$ povidone-iodine (Batticon; Adeka, Istanbul, Turkey), a lid speculum was positioned to keep the eyes open. The infants were held by experienced nurses throughout the procedure and $0.625 \mathrm{mg}(0.025 \mathrm{~mL})$ intravitreal bevacizumab (Altuzan; F. Hoffmann-La Roche, Basel, Switzerland) was injected I $\mathrm{mm}$ posterior to the limbus using a 30-gauge needle. After the injection, retinal artery perfusion was controlled using indirect ophthalmoscopy. All of the procedures were carried out by the same surgeon (Dr. SGÇ). A topical antibiotic eye drop was prescribed to be applied 4 times a day for 5 days following the procedure.

Laser photocoagulation (LPC) was performed under gen- eral anesthesia with an $810 \mathrm{~nm}$ diode laser indirect ophthalmoscope (Oculight SL; Iridex Corp., Mountain View, CA, USA). Laser ablation was applied to the entire avascular retina between the disease border and the ora serrata in a near confluent pattern. Topical antibiotic and steroid eye drops were prescribed to use for 7 days following the laser treatment.

After the procedure, the infants were examined at I day, I week, 3 weeks, and every month after the treatment. Subsequent control examinations occurred according to disease status and vascularization. The patients were monitored until full vascularization of the retina was observed.

Fluorescein angiography (FA) was performed using the Retcam3 (Clarity Medical Systems, Pleasanton, CA, USA). When incomplete or abnormal vascularization was seen with indirect ophthalmoscopy after 60 weeks gestational age (GA), FA was performed on patients whose parents approved the procedure. The FA procedure was carried out in an operating room under intravenous sedation. The examination included a bolus of $10 \%$ fluorescein solution (Alcon, Geneva, Switzerland) administered intravenously and a dose of $0.1 \mathrm{~mL} / \mathrm{kg}$ body weight. Photographs were recorded in the early, middle, and late phases. All of the treated infants were carefully observed by a treating neonatologist. In addition, a systemic evaluation was conducted during and after the FA procedure. Vascular abnormalities observed with FA were classified according to the Lepore et al. study (10).

In order to evaluate the subsequent overall development of ROP patients who underwent anti-VEGF treatment, the Denver II Developmental Test was administered to evaluate neurodevelopment in the patients over 2 years of age who had been treated with IVB. The Denver II test was administered by a pediatrician using the latest version developed specifically for Turkish children. A total of I 34 different tasks were evaluated for gross motor, fine motor, expressive language, and personal social skills. Each patient's performance was monitored and recorded for each task in order to compare them with age-based standards (II). The statistical analyses were performed using IBM SPSS Statistics for Windows, Version 22.0 software (IBM Corp., Armonk, NY, USA). The Kruskal-Wallis and Mann-Whitney $U$ tests were used for group analysis. A value of $p<0.05$ was accepted as statistically significant.

\section{Results}

In all, 38 eyes of 19 infants were included in the study and the mean length of follow-up was $32.2 \pm 4.3$ months (range: $24-$ 36 months). IVB treatment was performed on 9 infants with APROP disease (Group I), 6 infants with zone I disease at any stage with plus disease (Group 2), and 4 infants with stage 2-3 disease with plus disease in zone II (Group 3). The mean 
birth weight in the study group was $733 \pm 101 \mathrm{~g}$ (range: 600 $950 \mathrm{~g}$ ) in Group I, 723 $\pm 105 \mathrm{~g}$ (range: 610-850 g) in Group 2, and 1605 $\pm 103 \mathrm{~g}$ (range: 1460-I700 g) in Group 3. The mean GA was $26.5 \pm 1.23$ weeks (range: $25-29$ weeks) in Group I and 26.5 \pm I.76 weeks (range: $25-30$ weeks) in Group 2, and $32.2 \pm 0.82$ weeks (range: $31-33$ weeks) in Group 3. The mean GA at the time of the injection was $32.2 \pm 0.44$ weeks (range: $32-33$ weeks) in Group I, $32.5 \pm 0.83$ weeks (range: 32-34 weeks) in Group 2, and 38.5 \pm 2.06 weeks (range: 354 I weeks) in Group 3. No statistically significant difference was found in the GA, birth weight, or treatment weeks between Group I and Group 2 ( $p>0.05$ ); however, a significant difference was observed in Group $3(p=0.02)$.

Complete retinal vascularization (via indirect ophthalmoscopic examination) was observed in 24 eyes (4 APROP; 12 zone I, stage 3 disease; 8 zone II, stage 3 disease) treated with a single dose of bevacizumab therapy and required no additional treatment. Completion of vascularization was seen at a mean of 59.5 \pm 6.3 weeks (range: $55-64$ weeks) in Group I, 64.2 \pm 2.8 weeks (range: 6I-68 weeks) in Group 2, and $51.75 \pm 2.4$ weeks (range: $48-55$ weeks) in Group 3. Recurrence developed in 12 eyes of 6 infants with previously diagnosed APROP disease with a mean observation at $12.8 \pm 2.4$ weeks (range: 9-16 weeks) after the first treatment. Recurrence was viewed as a classic disease (not APROP or atypical ROP) and LPC was performed on those patients (Table I).

Complete retinal vascularization was not established in either eye of $I$ infant with APROP disease and recurrence occurred at the 83rd week. Nonetheless, in both the recurrence and non-recurrence groups, good anatomical and

Table I. Results of the current study

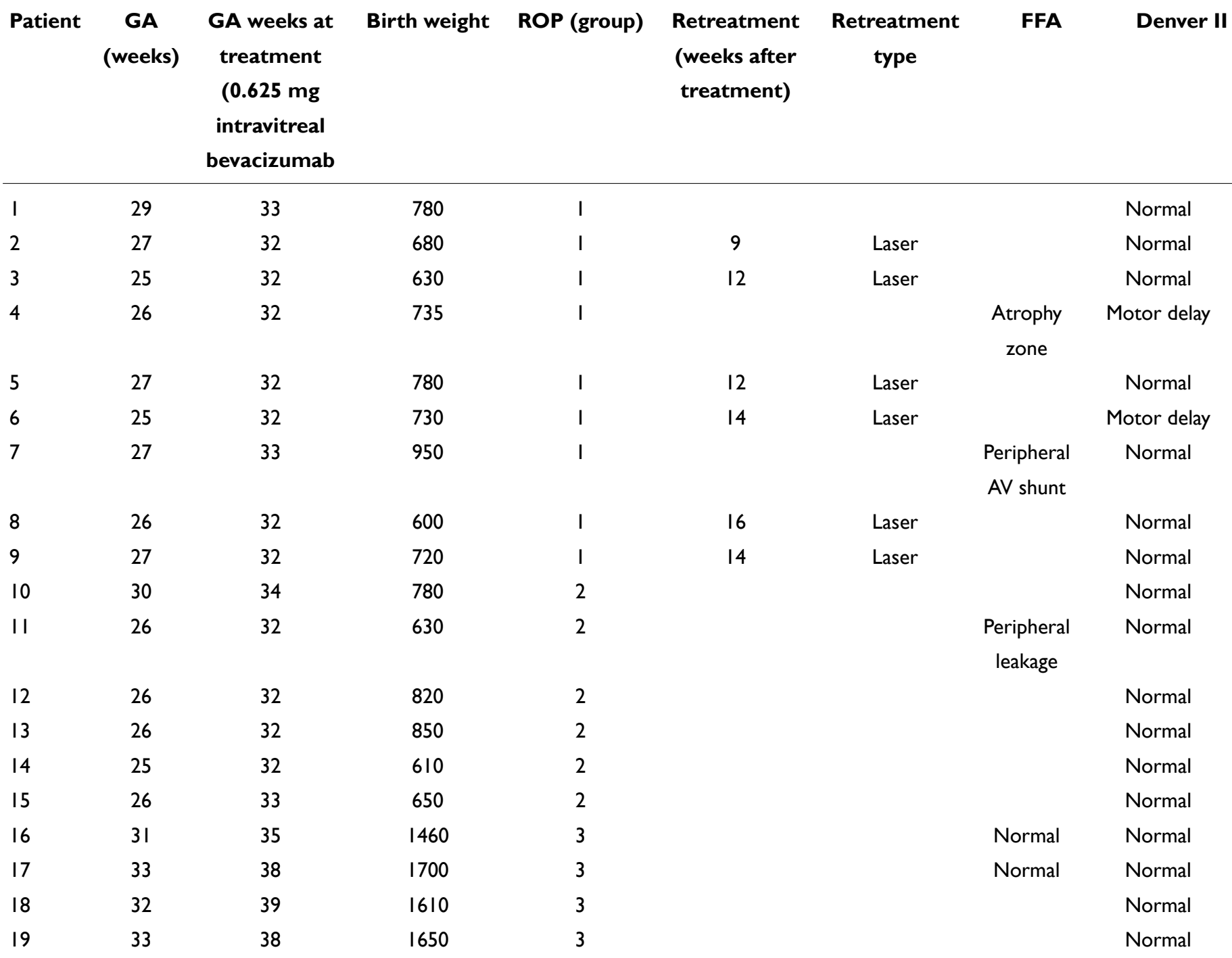


functional results were obtained. No complications, such as cataract or endophthalmitis, were detected.

An increase in the cup-to-disc (C/D) ratio was observed in I infant diagnosed with APROP within I week of the IVB injection. Intraocular pressure measurements were performed with a tonometer (Tono-Pen; Bio-Rad, Hercules, CA, USA), and the results were $16 \mathrm{Mm} \mathrm{Hg}$ in the right eye and $28 \mathrm{Mm} \mathrm{Hg}$ in the left eye. Anti-glaucoma drops (50\% diluted dorzolamide/timolol combination) were prescribed following the measurement. After 4 weeks of treatment with the anti-glaucoma drops, it was observed that the progressive axial length had ceased to increase and the high intraocular pressures had decreased to $16 \mathrm{Mm} \mathrm{Hg}$ and $22 \mathrm{Mm} \mathrm{Hg}$, in the right and left eye, respectively. Elevated intraocular pressure was not observed during the monthly follow-up visits. At the end of the sixth month, the use of anti-glaucoma drops was discontinued. Regression was also observed in the axial length and in the C/D ratio (Fig. I).

FA was performed on 5 patients who had received an anti-VEGF injection when the parents approved the procedure. In 2 patients from Group 3, the FA assessment was normal (Fig. 2). In I, one patient, areas of chorioretinal atrophy and macular hyperfluorescence were detected, and in another patient, peripheral avascular retina areas and vascular leakage were noted (Figs. 3, 4). Arteriovenous shunt formation was observed in I, one of the patients who underwent the FA procedure (Fig. 5).

The results of the Denver II Developmental Test administered to 19 children revealed that the test outcomes proved consistent with the corrected age of the infants. Two patients showed a developmental delay in gross motor tasks while demonstrating normal development in fine motor, personal social, and expressive language skills. Both patients with a motor delay had a grade 3 intracranial hemorrhage

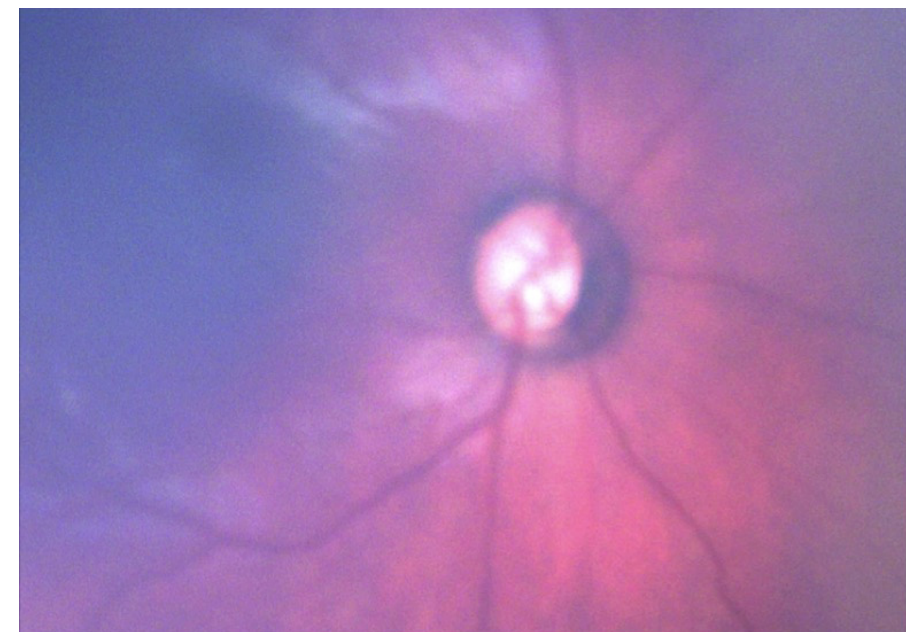

Figure I. Increased C/D ratio in one patient with APROP disease after anti-VEGF injection (68 weeks of age). and ventriculoperitoneal shunt surgery had been performed during neonatal unit care. The corrected age of the Denver II test was $25.2 \pm I .4$ months (range: $24-27$ months) (Table I).

\section{Discussion}

Our aim in this study was to investigate the safety and efficacy of IVB therapy in the treatment of APROP disease; zone I, any stage, with plus disease; and zone II, stage 2-3, with plus disease.

LPC is still the gold standard for ROP treatment but it has been demonstrated that the success rate of laser treatment might be low in zone I disease $(3,9,30)$ and different strategies are warranted (12). Subsequent studies indicated that if LPC is initially performed on the avascular retina and

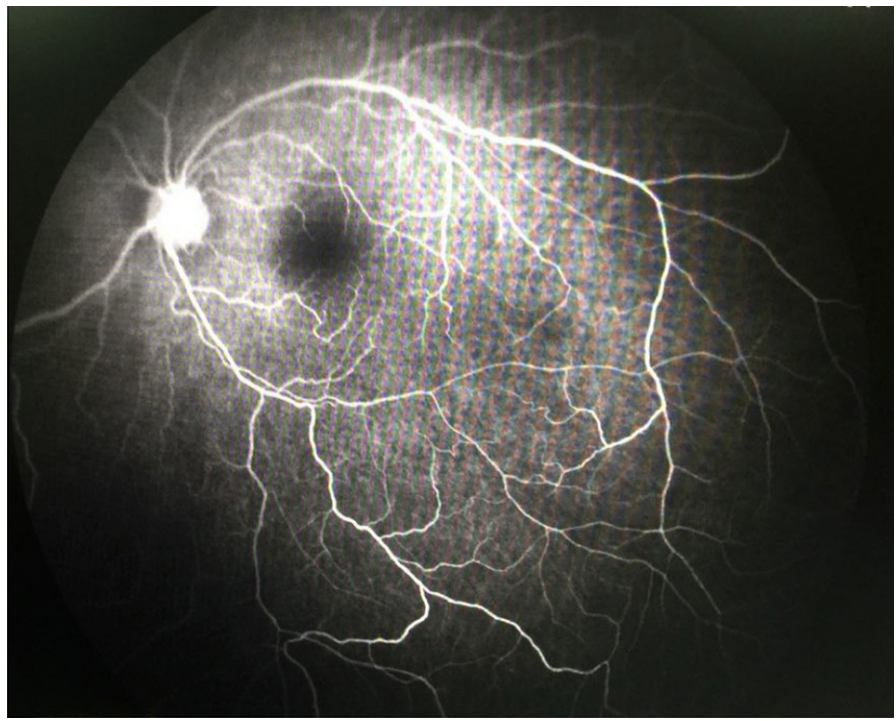

Figure 2. Normal FA image of a patient in group 3 (66 weeks of age).

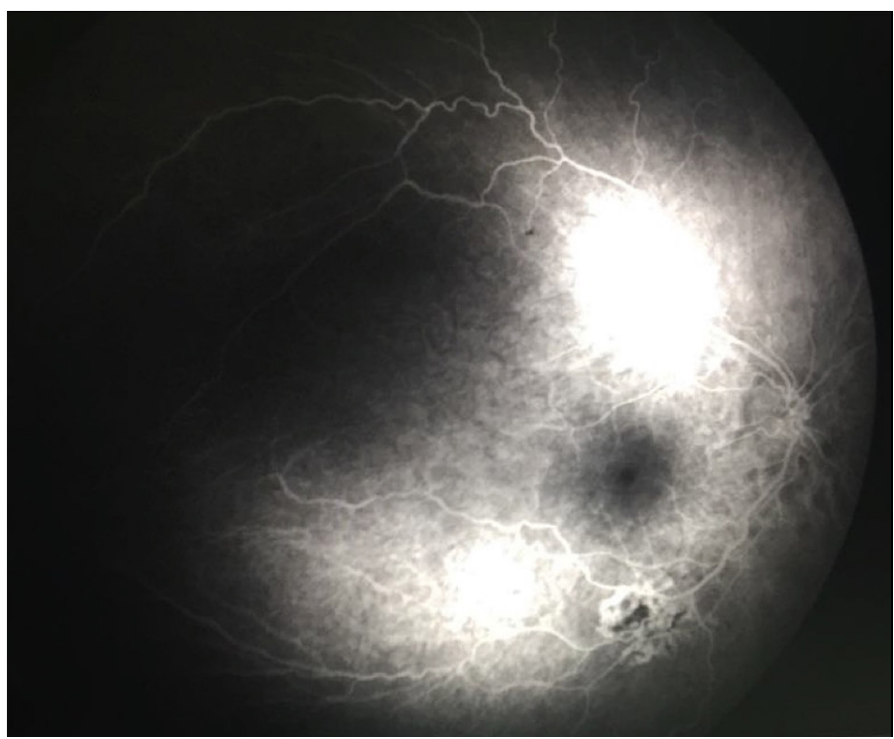

Figure 3. Chorioretinal atrophy area and persistent vascular tortuosity in a group I patient (69 weeks of age). 


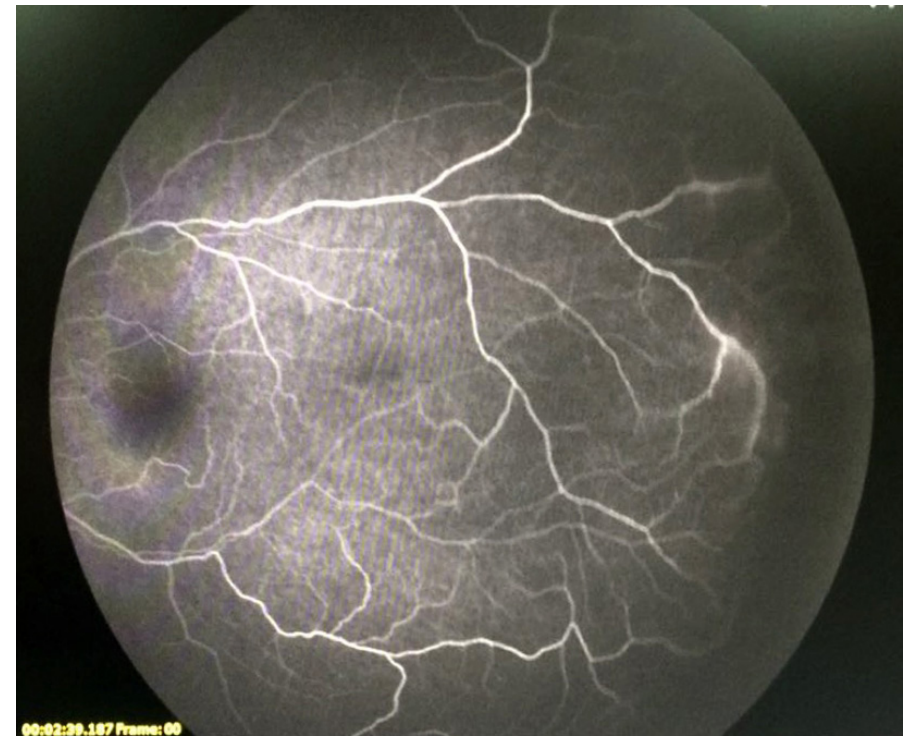

Figure 4. Peripheral vascular leakage in group 2 patients (65 weeks of age).

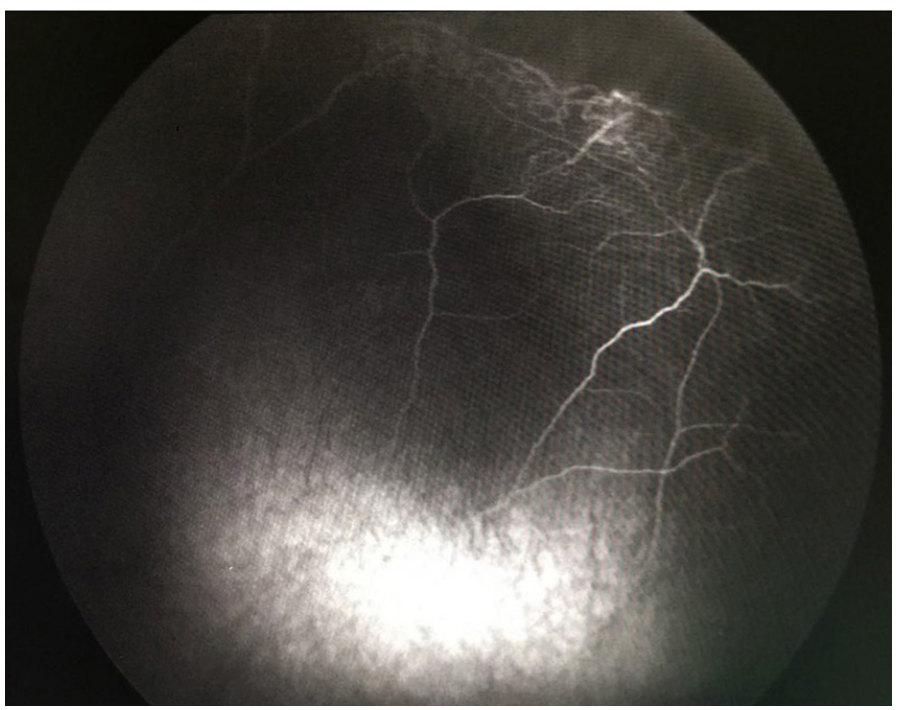

Figure 5. Peripheral av shunting in a group I patient (66 weeks of age).

then on the flat neovascularization region a week later, it caused less hemorrhage and fibrous component formulation. While LPC might result in a permanent effect in ROP treatment, the transient increase in VEGF after destruction of the avascular retina and the decrease in VEGF concentration in the vitreous after 2-3 weeks of treatment may cause disease progression in critical cases $(4,12)$. APROP disease, in particular, is characterized by vascular changes like flat neovascularization and intraretinal shunt formations, and retinal detachment may evolve rapidly $(3,12)$. Furthermore, the influence of peripheral visual field loss and the possibility of the enlargement of laser spots is not known in these patients, who have a long life expectancy (I3).

IVB has been widely used in other retinal diseases and was initially considered as a complementary treatment to LPC. Monotherapy techniques were developed when successful results were obtained with IVB alone in patients who were not able to undergo laser treatment due to unfavorable retinal imaging (14).

IVB is thought to cause regression of neovascularization by reducing the VEGF concentration in the vitreous, which creates the conditions for normal retinal vascularization (4).

When IVB treatment is used as monotherapy in ROP, the first problem is the transient effect. If the VEGF blocking effect of the drug decreases before the completion of vascularization, the disease can recur and additional injections or laser treatment may be required (20). There is still no consensus on the choice of the right anti-VEGF drug, the dosage, or the timing of an injection (15).

Lorenz et al. (16) treated 17 pre-term infants with severe ROP using IVB $(0.312 \mathrm{mg}$ in $0.025 \mathrm{~mL}$ per eye) and the results indicated that acute ROP regressed in 19 of the 27 monitored eyes $(70 \%)$ with a single injection. The regression rate was $100 \%$ ( 9 eyes) and $80 \%$ (8 eyes) in posterior zone II and zone I eyes, respectively, while it proved to be $25 \%$ (2 eyes) in aggressive posterior ROP eyes. The study population consisted of infants with APROP at a GA of $234 / 7$ weeks \pm 7 days and a mean birth weight of $58 \mathrm{I} \pm \mathrm{I} 13 \mathrm{~g}$. The success rate in our research proved $100 \%$ in the APROP group. This variation may be explained by the higher dosage we applied or by the heavier weight of the patients included in our study. Similarly, Ekici et al. (3I) reported a 100\% regression rate in APROP patients with an aflibercept injection. Mintz-Hitner et al. (4) also observed successful treatment with $0.625 \mathrm{mg}$ IVB in I infant. APROP treatment remains a difficult challenge, however. The results vary across a number of studies.

In our study, 4 infants categorized as stage 3 with plus disease in zone II who received an IVB injection demonstrated completed vascularization without any complication. Wu et al. (I7) injected IVB 4 I eyes of 23 patients and they observed regression after only $I$ dose of bevacizumab in 37 of $4 \mathrm{I}$ eyes with stage 3 ROP. LPC was used to treat the remaining 4 eyes with recurrence in order to achieve ROP regression. Menke et al. (I8) observed complete retinal vascularization 6 months after intravitreal ranibizumab monotherapy in ROP infants with zone II, stage 3, and plus disease in 6 eyes of 4 patients.

In a study conducted by Mintz et al. (19), no recurrence was observed among 29 of 31 zone I patients who had received an IVB injection. As for the structural result, macular dragging was seen in I patient.

Hu et al. (20) retrospectively reviewed the data of 17 eyes of 9 patients who had experienced recurrence of ROP following an initial treatment with IVB monotherapy and found a mean recurrence time of 14.4 weeks. In our study, the mean time to recurrence was 12.8 weeks. Hu et al. treated 
eyes with recurrent ROP disease after an IVB injection with a second bevacizumab injection in 4 eyes, LPC in 12 eyes, and surgical retinal detachment repair in 5 eyes. We preferred to use LPC on the avascular retina region of 6 infants with recurrent disease.

In a study by $\mathrm{Hu}$ et al., study, the maximum age of patients with recurrence requiring treatment was 69 weeks postmenstrual age (PMA). In the BEAT-ROP study (Bevacizumab Eliminates the Angiogenic Threat for Retinopathy of Prematurity), the primary outcome endpoint for the assessment of recurrence was 54 weeks PMA (19). Although the latest recurrence occurred at 48 weeks PMA, persistent avascular retina areas in zone III were seen in I eye of I infant after the age of 83 weeks. Yetik et al. (25) observed regression with a single IVB injection in $95.4 \%$ of their study group comprised of 238 eyes of 122 infants with threshold APROP disease. They applied a second injection to II eyes with recurrence of the disease, and then a third injection to 4 eyes with a second recurrence. Eventually, all of the eyes in that study group demonstrated full retinal vascularization without any complication. In our study, an avascular section spanning an area of $3 \mathrm{~mm}$ (larger than 2 optic disc diameters) from the ora serrata was observed in I patient. Tahija et al. (26) reported that among 20 eyes with ROP treated with IVB as monotherapy, peripheral avascular areas of more than 2 disc diameters were present up to 4 years after treatment in more than $50 \%(\mathrm{n}=\mathrm{II}$ eyes). Of those $\mathrm{II}$ eyes, 9 had fluorescein leakage at the vascular-avascular junction. In our study, I patient had vascular leakage. We believe that more fluorescein leakage and avascular areas may have been detected if FA had been performed in more patients. In addition to $F A$, assessment of the peripheral avascular region and vascular leakage is essential regarding late recurrence.

Lorenz et al. (16) observed that FA anomalies have been reported, such as dye leakage at the site of active ROP, abnormal vascular branching, circumferential vessels ("naked" arteriovenous shunt), hyperfluorescent lesion, capillary tuft formation, areas of hypofluorescence, periarteriolar loss of capillary bed macula, absence of foveal avascular zone, hypoperfusion, and hyperfluorescence due to leakage. In our study, we had similar findings of chorioretinal atrophy and macular hyperfluorescence, peripheral avascular retina areas, vascular leakage, and arteriovenous shunt formation. The FA findings of 2 members of Group 3 were evaluated as normal. Two of the children in Group 3 were bigger and heavier; there was less avascular retinal space to complete. Group 3 completed vascularization earlier than other groups at a mean of $51.75 \pm 2.4$ weeks (range: $48-55$ weeks), while the mean was $59.5 \pm 6.3$ weeks (range: $55-64$ weeks) in Group I and $64.2 \pm 2.8$ weeks in Group 2.

Although intravitreal therapy in premature infants ap- pears to be safe, important concerns remain regarding the escape of the anti-VEGF drug from the vitreous to the systemic circulation and the quantity of an effective dose. The volume of the vitreous in adults is $4 \mathrm{~mL}$, while it is $1 \mathrm{~mL}$ in infants. Such differences between adults and infants have generated discussion about the safe and appropriate dose, but a commonly well-accepted dose in infants is half of the adult dose $(0.025-0.03 \mathrm{~mL})(2 \mathrm{I}, 25)$. VEGF concentration in the systemic circulation is critical in premature organogenesis. Sato et al. (2I) measured the serum concentration of bevacizumab and VEGF before and after an intravitreal injection of $0.25 \mathrm{mg}$ or $0.5 \mathrm{mg}$ bevacizumab in II infants. They observed a significant negative correlation between the serum concentration of bevacizumab and the VEGF dose. However, one of the significant limitations of the study was that they applied LPC on the peripheral avascular retina before the administration of IVB. The LPC procedure can break down the retinal barrier. During the period when development is in progress, neurological tests can be useful to observe the effects of these medications. In our study, normal neurological development was observed in 17 of the 19 infants treated with anti-VEGF; gross motor delay was detected in 2 infants. Martinez-Garcia et al. (6) reported that the majority of 7 patients injected with $0.625 \mathrm{mg}$ IVB showed standard neurodevelopmental scores according to the Bayley Scale of Infant Development. In another study of 125 infants in which Morin et al. (7) compared the use of laser and bevacizumab, greater odds of neurodevelopmental disabilities were observed in the group treated with bevacizumab at the end of an 18-month period. Martinez-Castellanos et al. (22) also analyzed the effect of IVB, using a dosage of $1.25 \mathrm{mg}$ in 13 patients, and they found that only I patient showed neurodevelopmental delay at the end of a 5-year period. Our outcomes are similar. We observed disabilities in 2 patients. These children with a motor delay may be able to catch up with their peers as they age, or the condition may persist, with the possibility of additional health problems. Developmental delays are frequently seen in children born prematurely, and may continue until the pre-school or school age. (23) The developmental delay in our cases was probably due to prematurity, rather than the systemic effect of anti-VEGF drugs. In this study, we administered the Denver II test at a minimum of 2 years of age to reduce the prematurity effect. Weight and the number of gestational weeks may result in a larger immature retinal surface, and an anti-VEGF injection may induce systemic transition and cause more developmental delay. The Denver II scale is useful for the evaluation of neurodevelopment, but for nutritional or systemic effects, other specific tests should also be employed. We did not detect any systemic problems in our patients, including the respiratory system. Respiratory disease is a significant source of morbidity and mortality in 
premature infants, and VEGF is also known to play an essential role in alveolar maturation (24).

Anti-VEGF drugs administered intravitreally to treat ROP disease can trigger fibrovascular proliferation and acute contraction of the retina, and these contractions can cause retinal detachment. In a case report submitted by Honda et al. (27), acute contraction of the proliferative membrane was observed, resulting in funnel-like retinal detachment in I eye of an infant who had received an IVB injection due to stage IVA ROP disease. Serious adverse events, such as a macular hole, rhegmatogenous retinal detachment, bilateral progressive vascular attenuation, perivascular exudation, and optic atrophy have also been reported after an IVB injection for ROP (28). In our study, we observed a progressive axial length increase and a rise in the intraocular pressure in I eye without any sign of congenital glaucoma. This complication was thought to occur due to an accumulation of bevacizumab molecules in the trabecular meshwork, and it was treated with anti-glaucoma drops. Additionally, protein aggregates and silicone oil microdroplets can accumulate in the bevacizumab syringes, and this accumulation can occlude the trabecular meshwork, leading to a rise in the intraocular pressure (29). We think that this elevation persists until the molecule is absorbed; it may be transient or permanent according to the organization of the trabecular meshwork.

The limitations of our study include the small number of patients, the application of FA on only some of patients, and a short follow-up period for neurological development. This research was not designed as a drug safety study.

In conclusion, an IVB injection is an effective treatment modality for infants with ROP disease. Good anatomical, functional, and neurodevelopmental results can be obtained in most cases. Higher rates of recurrence or persistence are more likely in APROP patients; however FA can be used to detect vascular malformation. The Denver II and other tests are important tools for neurodevelopmental assessment.

In conclusion, additional studies are required to further determine the safety of anti-VEGF drugs in ROP disease and the potential side effects.

\section{Disclosures}

Ethics Committee Approval: 20I I-KAEK-25 2018/09-06.

Peer-review: Externally peer-reviewed.

Conflict of Interest: None declared.

Authorship Contributions: Involved in design and conduct of the study (SGC, IP); preparation and review of the study (SGC, MTC); data collection (SGC, MTC); and statistical analysis (SGC, MTC, IP).

\section{References}

I. Howson CP, Kinney MV, McDougall L, Lawn JE; Born Too Soon Preterm Birth Action Group. Born too soon: preterm birth matters. Reprod Health 2013;10:SI. [CrossRef]

2. No authors listed. Multicenter Trial of Cryotherapy for Retinopathy of Prematurity Preliminary Results. Arch Ophthalmol 1988;106:47|-9. [CrossRef]

3. Vander JF, McNamara JA, Tasman W, Brown GC. Revised indications for early treatment of retinopathy of prematurity. Arch Ophthalmol 2005; 123:406-7. [CrossRef]

4. Mintz-Hittner HA, Kuffel RR. Intravitreal injection of bevacizumab (Avastin) for treatment of stage 3 retinopathy of prematurity in zone I or posterior zone II. Retina 2008;28:83 I-8.

5. Hillier RJ, Connor AJ, Shafiq AE. Ultra-low-dose intravitreal bevacizumab for the treatment of retinopathy of prematurity: a case series. Br J Ophthalmol 2018;102:260-4. [CrossRef]

6. Martínez-García SM, Hernández-Da Mota SE, Rubio-Rangel A, Rojas-Flores I, Vieyra-López ME, Martínez-Castellanos MA, et al. Neurodevelopment in patients with retinopathy of prematurity treated with intravitreal bevacizumab. Case series Cir Cir 20। 7;85:478-84. [CrossRef]

7. Morin J, Luu TM, Superstein R, Ospina LH, Lefebvre F, Simard $\mathrm{MN}$, et al. Canadian Neonatal Network and the Canadian Neonatal Follow-Up Network Investigators. Neurodevelopmental Outcomes Following Bevacizumab Injections for Retinopathy of Prematurity. Pediatrics 2016;137.e20153218.

8. International Committee for the Classification of Retinopathy of Prematurity. The International Classification of Retinopathy of Prematurity revisited. Arch Ophthalmol 2005; 123:99I-9.

9. Good WV; Early Treatment for Retinopathy of Prematurity Cooperative Group. Final results of the Early Treatment for Retinopathy of Prematurity (ETROP) randomized trial. Trans Am Ophthalmol Soc 2004; 102:233-50.

10. Lepore D, Quinn GE, Molle F, Baldascino A, Orazi L, Sammartino $M$, et al. Intravitreal bevacizumab versus laser treatment in type I retinopathy of prematurity: report on fluorescein angiographic findings. Ophthalmology 2014;121:2212-9.

I I. Anlar B, Yalaz K. Denver II Gelişimsel Tarama Testi. Türk çocuklarına uyarlanması ve standardizasyonu HÜTF Ped. Ankara: Nöroloji Bilim Dalı; 1995. p. I-43.

12. Kim R, Kim YC. Posterior pole sparing laser photocoagulation combined with intravitreal bevacizumab injection in posterior retinopathy of prematurity. J Ophthalmol 20I4;20I4:257286.

13. Maeshima K, Utsugi-Sutoh N, Otani T, Kishi S. Progressive enlargement of scattered photocoagulation scars in diabetic retinopathy. Retina 2004;24:507-II. [CrossRef]

14. Law JC, Recchia FM, Morrison DG, Donahue SP, Estes RL. Intravitreal bevacizumab as adjunctive treatment for retinopathy of prematurity. J AAPOS 2010;14:6-10. [CrossRef]

15. Wallace DK, Dean TW, Hartnett ME, Kong L, Smith LE, Hubbard GB, et al. Pediatric Eye Disease Investigator Group. A Dosing Study of Bevacizumab for Retinopathy of Prematurity: Late Recurrences and Additional Treatments. Ophthalmology 20|8; I 25:|96|-6. [CrossRef] 
16. Lorenz B, Stieger K, Jäger M, Mais C, Stieger S, Andrassi-Darida M. Retinal Vascular Development with $0.312 \mathrm{mg}$ Intravitreal Bevacizumab to Treat Severe Posterior Retinopathy of Prematurity: A Longitudinal Fluorescein Angiographic Study. Retina 2017;37:97-III. [CrossRef]

17. Wu WC, Yeh PT, Chen SN, Yang CM, Lai CC, Kuo HK. Effects and complications of bevacizumab use in patients with retinopathy of prematurity: a multicenter study in taiwan. Ophthalmology 201 I; I 18:176-83. [CrossRef]

18. Menke MN, Framme C, Nelle M, Berger MR, Sturm V, Wolf S. Intravitreal ranibizumab monotherapy to treat retinopathy of prematurity zone II, stage 3 with plus disease. BMC Ophthalmol 20I5; I5:20. [CrossRef]

19. Mintz-Hittner HA, Kennedy KA, Chuang AZ; BEAT-ROP Cooperative Group. Efficacy of intravitreal bevacizumab for stage 3+ retinopathy of prematurity. N Engl J Med 201 I;364:603-I5.

20. Hu J, Blair MP, Shapiro MJ, Lichtenstein SJ, Galasso JM, Kapur R. Reactivation of retinopathy of prematurity after bevacizumab injection. Arch Ophthalmol 2012;130:1000-6. [CrossRef]

2I. Sato T, Wada K, Arahori H, Kuno N, Imoto K, Iwahashi-Shima $C$, et al. Serum concentrations of bevacizumab (avastin) and vascular endothelial growth factor in infants with retinopathy of prematurity. Am J Ophthalmol 2012;153:327-33. [CrossRef]

22. Martínez-Castellanos MA, Schwartz S, Hernández-Rojas ML, Kon-Jara VA, García-Aguirre G, Guerrero-Naranjo JL, et al. Long-term effect of antiangiogenic therapy for retinopathy of prematurity up to 5 years of follow-up. Retina 2013;33:329-38.

23. Howe TH, Sheu CF, Hsu YW, Wang TN, Wang LW. Predicting neurodevelopmental outcomes at preschool age for children with very low birth weight. Res Dev Disabil 2016;48:231-4I.

24. Thébaud B. Angiogenesis in lung development, injury and repair: implications for chronic lung disease of prematurity. Neonatology 2007;91:291-7. [CrossRef]
25. Yetik H, Gunay M, Sirop S, Salihoglu Z. Intravitreal bevacizumab monotherapy for type-I prethreshold, threshold, and aggressive posterior retinopathy of prematurity - 27 month follow-up results from Turkey. Graefes Arch Clin Exp Ophthalmol 2015;253:1677-83. [CrossRef]

26. Tahija SG, Hersetyati R, Lam GC, Kusaka S, McMenamin PG. Fluorescein angiographic observations of peripheral retinal vessel growth in infants after intravitreal injection of bevacizumab as sole therapy for zone I and posterior zone II retinopathy of prematurity. Br J Ophthalmol 2014;98:507-12. [CrossRef]

27. Honda S, Hirabayashi H, Tsukahara Y, Negi A. Acute contraction of the proliferative membrane after an intravitreal injection of bevacizumab for advanced retinopathy of prematurity. Graefes Arch Clin Exp Ophthalmol 2008;246:106I-3. [CrossRef]

28. Jalali S, Balakrishnan D, Zeynalova Z, Padhi TR, Rani PK. Serious adverse events and visual outcomes of rescue therapy using adjunct bevacizumab to laser and surgery for retinopathy of prematurity. The Indian Twin Cities Retinopathy of Prematurity Screening database Report number 5. Arch Dis Child Fetal Neonatal Ed 2013;98:F327-F33. [CrossRef]

29. Liu L, Ammar DA, Ross LA, Mandava N, Kahook MY, Carpenter JF. Silicone oil microdroplets and protein aggregates in repackaged bevacizumab and ranibizumab: effects of long-term storage and product mishandling. Invest Ophthalmol Vis Sci 201 I;52:1023-34. [CrossRef]

30. Kychenthal A, Dorta P, Katz X. Zone I retinopathy of prematurity: clinical characteristics and treatment outcomes. Retina 2006;26:SI I-S5. [CrossRef]

3I. Ekinci DY, Çelik K. Comparison of the Efficacy Between Intravitreal Aflibercept and Laser Photocoagulation in the Treatment of Retinopathy of Prematurity. J Pediatr Ophthalmol Strabismus 2020;57:54-60. [CrossRef] 